\title{
Adaptive optics imaging of a stellar occultation by Titan
}

\section{Antonin H. Bouchez, Michael E. Brown, Mitchell Troy, Rick S. Burruss, Richard G. Dekany, et al.}

Antonin H. Bouchez, Michael E. Brown, Mitchell Troy, Rick S. Burruss, Richard G. Dekany, Robert A. West, "Adaptive optics imaging of a stellar occultation by Titan," Proc. SPIE 4839, Adaptive Optical System Technologies II, (7 February 2003); doi: 10.1117/12.459463

Event: Astronomical Telescopes and Instrumentation, 2002, Waikoloa, Hawai'i, United States 


\title{
Adaptive optics imaging of a stellar occultation by Titan
}

\author{
Antonin H. Bouchez ${ }^{a}$, Michael E. Brown ${ }^{a}$, Mitchell Troy ${ }^{b}$, \\ Rick S. Burruss ${ }^{c}$, Richard G. Dekany ${ }^{d}$, Robert A. West ${ }^{b}$ \\ ${ }^{a}$ Div. of Geol. and Planetary Sci., California Inst. of Technology, Pasadena, CA 91125, USA \\ ${ }^{b}$ Jet Propulsion Laboratory, California Inst. of Technology, Pasadena, CA 91109, USA \\ ${ }^{c}$ Palomar Observatory, 35899 Canfield Rd., Palomar Mountain, CA 92060, USA \\ ${ }^{d}$ Caltech Optical Observatories, California Inst. of Technology, Pasadena, CA 91125, USA
}

\begin{abstract}
We present resolved images of the occultation of a binary star by Titan, recorded with the Palomar Observatory adaptive optics system on 20 December 2001 UT. These constitute the first resolved observations of a stellar occultation by a small body, and demonstrate several unique capabilities of diffraction-limited imaging systems for the study of planetary atmospheres. Two refracted stellar images are visible on Titan's limb throughout both events, displaying scintillations due to local density variations. Precise relative astrometry of the refracted stellar images with respect to the unnocculted component of the binary allows us to directly measure their altitude in Titan's atmosphere. Their changing positions also lead to simple demonstration of the finite oblateness of surfaces of constant pressure in Titan's mid-latitude stratosphere, consistent with the only previous measurement of Titan's zonal wind field.
\end{abstract}

Keywords: Adaptive optics, occultation, Titan, Titan atmosphere

\section{INTRODUCTION}

Occultations provide one of the principal tool with which we can probe the atmospheres of solar system planets and satellites from the Earth. They can be used to derive temperature, pressure, and number density profiles, and sometimes to probe the opacity structure and zonal wind fields of the atmospheres on distant bodies (see the review by Elliot and Olkin ${ }^{1}$ ). Previous observations of occultations have generally been restricted to measuring the total planetary plus stellar flux as a function of time (the lightcurve), as an observer at a given location on Earth follows a chord across the planetary shadow.

The gradual drop of a star's lightcurve at the point of immersion into the body's atmosphere, and the subsequent rise at emersion, can be inverted to derive the local temperature and density profiles in the microbar pressure range. $^{2,3}$ The primary cause of the dimming is differential refraction, effectively defocusing light rays in the plane of the local density gradient along the ray's trajectory. Deeper within the planet or satellite's shadow, some starlight may still reach an observer on Earth, sampling the deeper atmosphere at locations on the limb where the density gradient lies in the plane defined by the star, the location on the limb, and the observer. For a perfectly spherical body, there will be two such points on opposite limbs, both possibly contributing to the observed stellar flux. Near the center of an oblate body's shadow, four locations on the limb may appear illuminated.

For observers whose path lies near enough to the shadow's geometric center, a "central flash" may be observed due to the coherent focusing of the starlight by multiple points on the planet's limb. Imagine the ideal case of a perfectly spherical atmosphere, observed from the midpoint of its shadow. Every point on the limb is then capable of refracting starlight to the observer, leading to a sudden increase in the total stellar intensity recorded. The central flash was first recorded in unresolved photometry of the occultation of $\epsilon$ Gem by Mars on 8 April 1976, where it was used to derived the mean opacity of the lower Martian atmosphere near the south pole (the region inferred to have been sampled by the magnified rays). ${ }^{4}$

Send correspondence to A.H.B.: E-mail: antonin@gps.caltech.edu, Telephone: 626-395-6961 
The appearance of two additional stellar images on an oblate body's limb when viewed from near the center of the shadow can be considered a limited form of a central flash. The position of refracted stellar images is sensitive to the shape of surfaces of constant pressure in the atmosphere, and can therefore be used to precisely determine its oblateness, a function of planetary rotation and of the zonal wind speeds. ${ }^{5}$ The only instance in which these multiple images have previously been individually resolved is during the occultation of $28 \mathrm{Sgr}$ by Saturn on 3 July 1989. Nicholson et. al. ${ }^{6}$ used the location of four stellar images detected intermittently on Saturn's limb to demonstrate that the mid-latitude tropospheric winds measured by cloud tracking decay with height to a global stratospheric flow whose speed is equal to the mean of the tropospheric values. The occultation of 28 Sgr by Titan several hours later on 3 July 1989 could not be similarly imaged do to Titan's small angular and the limitations of the telescopes available. Nevertheless, an intensive photometric observing campaign led to 19 separate lightcurves, which were synthesized to create a sparsely-sampled map of the stellar flux across Titan's shadow. ${ }^{7,8}$ The structure of the central flash recorded in these lightcurves was then modeled to provide a first estimate the zonal winds in Titan's lower stratosphere. ${ }^{7}$

The availability of adaptive optics (AO) and rapid-readout imaging detectors on large telescopes has created exciting new opportunities for the observation of stellar occultations by solar system bodies. Given an event with the appropriate geometry, the photometry and position of multiple refracted stellar images can now be separately measured for occultations by planets and satellites previously studied only with unresolved photometry. This allows atmospheric properties such as the zonal wind patterns and the opacity at millibar pressure levels on these bodies to be determined with unprecedented spatial detail from the Earth.

On 20 December 2001, both components of the $\mathrm{m}_{\mathrm{V}}=13.8$ binary star system NV0435215+200905 were occulted by Titan in quick succession. The event was visible from North and South America and Hawaii, and the shadow centerline was predicted to run across the American Southwest and Southeast. Observations of the occultation were coordinated by L. Young, ${ }^{9}$ with at least 7 observatories participating in the campaign. It has provided a spectacular demonstration of the some of the capabilities of adaptive optics for the study of distant planetary atmospheres. In this paper we offer a first look at the sequence of resolved images of this occultation acquired at Palomar Observatory.

\section{OBSERVATIONS}

We observed the occultation of the binary star system NV0435215+200905 by Titan on 20 December 2001 (UT) with the JPL adaptive optics system ${ }^{10}$ coupled to the the Cornell-built PHARO near-infrared camera ${ }^{11}$ at the cassegrain focus of the $5 \mathrm{~m}$ Palomar Hale telescope. Titan $\left(\mathrm{m}_{\mathrm{V}}=8.14\right)$ acted as the reference source with which the phase aberrations induced by Earth-atmospheric turbulence were measured. The relative faintness of the occulted stars in the visible $\left(\mathrm{m}_{\mathrm{V}} \approx 13.8\right.$ combined $\left.^{9}\right)$ rendered them effectively invisible to the ShackHartmann wavefront sensor. The AO correction quality was optimized for the color and angular size of Titan by adjusting the locations to which the Shack-Hartmann spots were driven. All data were taken through a $\mathrm{K}$ ' filter $(1.945-2.296 \mu \mathrm{m})$ to maximize the $\mathrm{AO}$ correction quality and the photons collected from the very red stars $\left(\mathrm{m}_{\mathrm{K}}=10.61\right.$ combined). The Strehl ratio $S$, the ratio of the peak brightness measured on a point source to that of a diffraction-limited optical system, fluctuated in the range $0.21<S<0.47$ due to changing Earth-atmospheric conditions, with a median during the occultations of $S=0.33$.

Operating without a shutter, integration times on PHARO's $1024^{2}$ pixel HgTeCd HAWAII detector are determined by the time elapsed between readouts. The detector is clocked at a fixed rate and usually operated in a correlated double sampling mode, leading to a minimum integration time which is a function of the number of pixels read, and a time elapsed between integrations of slightly longer than one read (one read plus the time to reset the bias voltage). Balancing the requirements of a sufficiently wide field-of-view and the shortest possible integrations and dead time, we chose an $8 \times 4$ arcsec. $(320 \times 160$ pixels at 25.17 milliarcsec. per pixel $)$ field with an integration time of 437 millisecond (ms). The dead time between integrations was $443 \mathrm{~ms}$. Note that since the pixels are addressed sequentially, and the integration time is defined by the delay between two reads of a given pixel, the images returned are asynchronous, with a maximum delay between the integration midpoint across titan's disk of $13 \mathrm{~ms}$. 
Images were taken continuously from 4:48 UT to 5:58 UT, in sets of 256 with a gap of 4.083 seconds between sets, for a total of 4700 frames. Since Titan was the reference source tracked by the wavefront sensor, the satellite remained roughly fixed on the detector while the stars appeared to drift by from west to east. In each 4 second gap between exposure sets, we offset Titan by a fraction of an arcsecond eastward, to keep the field of view roughly centered on the midpoint between Titan and the stars.

\section{DATA REDUCTION}

The images were processed using standard near-infrared image reduction techniques, including the subtraction of a median dark frame, division by a mean of twilight sky observations (a map of pixel gains), and correction for insensitive pixels. However, the operation of the PHARO camera at such an unusually frenetic pace led to some electronic glitches which needed to be compensated for in the reduction process. The first 3-4 frames of each set of 256 exhibited a variable bias level, which appeared ramped along the slow-scan direction (north-south) of the detector. These frames were eliminated from the sets of dark exposures and twilight sky frames. The equivalent frames in the occultation imaging sequence were corrected by subtracting the median of pixels along the east and west margins of the frame from each row, then subtracting a similarly modified standard dark frame, before dividing by the pixel gain map. In a final step, the mean sky brightness measured far from Titan and the stars was subtracted from each frame.

The purpose of subsequent image processing was to subtract Titan's image from each frame, to allow faint refracted stellar images to be detected on the limb of the satellite. This is not trivial since the point-spread function (PSF) of the combined telescope/AO optical system varies from frame to frame. However, during the occultation of each star, the companion 1.5 arcsec. away served as a nearly ideal measure of the instantaneous PSF, greatly simplifying the task.

A deconvolved model of Titan's disk was calculate by running the blind deconvolution algorithm IDAC ${ }^{12}$ on 400 independent sets of 4 consecutive frames, in which the stars were well-separated from Titan's disk. Given $N$ images of the same field acquired with a varying PSF, IDAC iteratively determines the single deconvolved image and $N$ PSF maps which best fit the data in a least-squares sense, using prior information such as positivity and a band-limited PSF to break the degeneracy inherent in solving for $N+1$ quantities. The relative motion between Titan and the stars over 4 frames is only 0.34 pixels, allowing the field to be treated as unchanging in this context. The 400 independent deconvolved images of Titan were co-aligned by cross-correlation with a blank disk, and averaged to create our disk model. We did not use the PSFs calculated by IDAC in further processing, as they were typically somewhat broader than those measured directly from the companion star.

The extraction of the PSF of the star most distant from Titan in each frame also followed an iterative procedure, since the broad seeing-limited wings of Titan and the star were superimposed on one another. In each frame, a preliminary PSF was first extracted after the subtraction of Titan's mean radial profile. Each star was then precisely located and subtracted from the original image, using a non-linear least-squares minimization between the PSF model and the radially-subtracted image to solve for their relative offsets and flux ratios. Next we convolved our IDAC-derived model of Titan's disk by the preliminary PSF, and performed an identical leastsquares fit between this model image and the star-subtracted image, to determine the relative location and flux of Titan's disk. Finally, the convolved, shifted, and scaled model of Titan was subtracted from the original frame and the entire process repeated, until the extracted PSF showed no azimuthal asymmetries due to the incorporation of light from Titan. All image translations in this process were performed by adding a phase ramp to the imaginary part of the image's Fourier transform, then transforming back to the image domain. This technique preserves the spatial frequency content of the image, while allowing precise shifts of a fraction of a pixel.

The end result of this modeling and subtraction procedure was a set of 4700 Titan-subtracted frames, and an accurate PSF model for each. Figure 1 presents a single frame, both before and after subtraction. No coherent residual counts remain at Titan's location in the subtracted image. However, the random photon noise in the region of Titan's disk remains elevated by a factor of 4 over the background level of $6.2 \mathrm{DN}$ pixel $^{-1}$.

The accurate subtraction of Titan from each frame allowed us to precisely determine the relative locations and fluxes of the stars and refracted stellar images which remained. The technique used was identical to that 


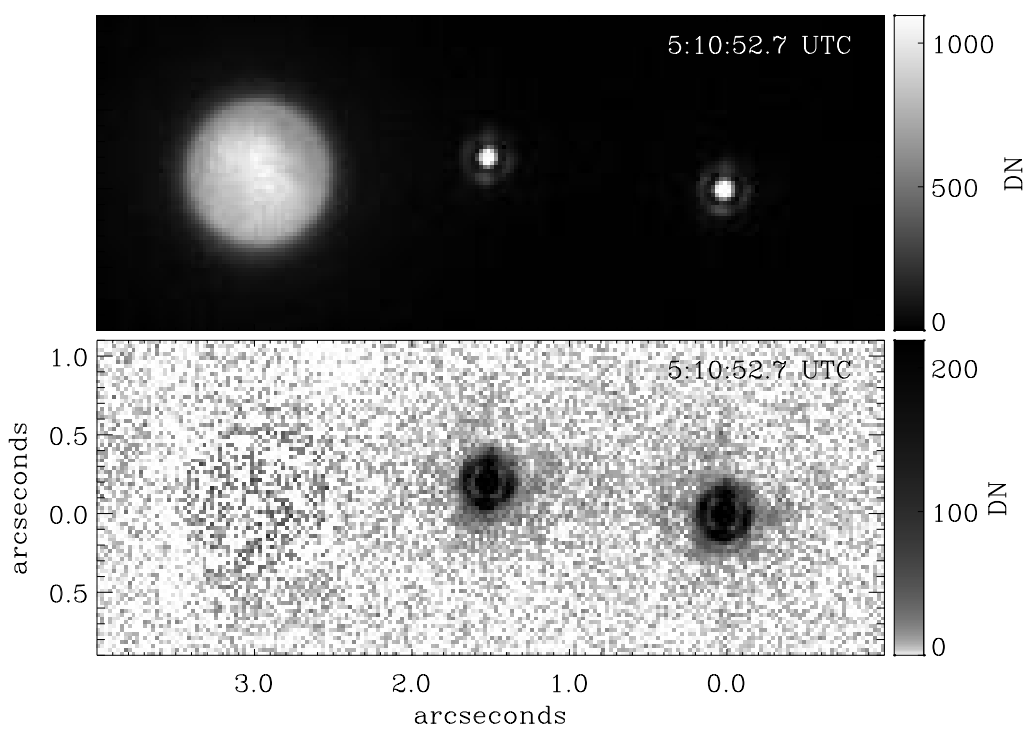

Figure 1. A single $437 \mathrm{~ms}$ integration from the occultation imaging sequence, 5 minutes before ingress of the eastern (left) star, shown before and after subtraction of Titan's image. The logarithmic grayscale of the lower panel highlights the absence of a coherent residual at Titan's location. The centroid of the brighter, western star defines the coordinate origin. Sharp albedo contrasts can be clearly seen on Titan's surface.

employed during the subtraction process, detailed above. In each frame, the square of the difference between the model PSF (that of the star furthest from Titan) and each other stellar image was minimized, solving for their relative offsets and flux ratios. The uncertainty in these quantities was determined by running Monte Carlo simulations for each frame, repeating the fit 100 times on synthetic datasets and adopting the $1 \sigma$ deviation of the results from the input parameters as the estimate of our uncertainty. Far from Titan, the relative position of the stars can be measured to better than 0.01 pixels $\left(2.5 \times 10^{-4}\right.$ arcsec. $)$, while the relative flux is determined to $1 \%$. For fainter refracted stellar images on Titan's limb, the uncertainty in relative position sharply increases to $\sim 0.15$ pixels in typical frames. Finally, the centroid of the PSF star served to correlate relative positions between frames, which introduced a nearly constant uncertainty of 0.04 pixels. We adopted the centroid of the brighter, western star as the origin of our reference coordinate system. The derived astrometric parameters of the occultations are summarized in Table 1.

Precise timing of each frame in the occultation imaging sequence was assured by synchronizing the datataking computer's system clock to Coordinated Universal Time (UTC) using Network Time Protocol software, accurate to better than $0.1 \mathrm{~s}$. The system time at the start of integration of each frame was written to the image header, rounded down to the nearest second. However, within each set of 256 frames the relative timing of the frames is known to $1 \mathrm{~ms}$. We therefore linearly interpolated the start times within each set, leading to a final timing accuracy of $0.1 \mathrm{~s}$. In this paper, we quote the time at which the integration of the first pixel in the frame began, rounded to the nearest $0.1 \mathrm{~s}$ UTC.

\section{RESULTS}

The first result of these observations was the immediate realization, as the star NV0435215+200905 crept into the field of view of the PHARO camera, that it was a clearly separated binary. Predictions of the occultation had assumed it to be a single star, and placed Palomar Observatory $80 \pm 250 \mathrm{~km}$ from the occultation centerline. ${ }^{9}$ Brief panic thus ensued as we feared that the two stars would pass to the north and south of Titan, while their center of light passed behind the satellite's disk. Happily this did not occur, and both stars were occulted in rapid succession, providing multiple samplings of Titan's atmosphere, a precise astrometric and flux reference, 
Table 1. Observational parameters

\begin{tabular}{|c|c|}
\hline east/west K' flux ratio & $0.90 \pm 0.01$ \\
\hline Separation & $1.518 \pm 0.004 \mathrm{arcsec}$. \\
\hline Position Angle & $83.27 \pm 0.01 \mathrm{deg}$. \\
\hline Midpoint of E star occultation & $5: 18: 22 \pm 1 \mathrm{~s}$ \\
\hline Impact parameter of E star & $0.096 \pm 0.002 \mathrm{arcsec}$. \\
& $560 \pm 15 \mathrm{~km}$ \\
\hline Midpoint of W star occultation & $5: 26: 05 \pm 1 \mathrm{~s}$ \\
\hline Impact parameter of W star & $0.106 \pm 0.002 \mathrm{arcsec}$. \\
& $630 \pm 15 \mathrm{~km}$ \\
\hline
\end{tabular}

and an invaluable measure of the instantaneous telescope/AO PSF. All results which follow will rely on relative measurements of one star with respect to the other.

To provide an overview of the phenomena observed during the occultation of NV0435215+200905 by Titan, we present in Fig. 2 two series of Titan-subtacted frames from the imaging sequence, beginning shortly before the immersion of each star and ending shortly after emersion. The frames were chosen to be spaced roughly equally in time, while avoiding those in which no stellar images were detected $(\sim 17 \%$ of the frames during each occultation). The frames have all been shifted to be fixed in the stellar reference frame. To supplement these stills, photometry of the near-limb refracted stellar image for each occultation is presented in Fig. 3. Our minimum detectable flux, $\sim 2 \%$ of the unocculted intensity of the brighter star, is shown with a dashed line. Below this limit, set by the photon noise which remains after the subtraction of Titan's disk, the least-squares fit between the PSF and the refracted stellar image would not converge.

The two stars exhibited somewhat different phenomena during the successive occultations, possibly due either to the slight difference in their impact parameters (their apparent distance of closest approach from Titan's geometric center) or the sampling of opposite hemispheres of Titan by the near- and far-limb refracted images of each. Upon ingress and egress, both stars underwent large fluctuations in brightness on a timescale shorter than our sampling rate of $880 \mathrm{~ms}$, though this effect was much more pronounced in the occultation of the western star, which passed south of Titan's center. This well-known phenomenon is caused by local density variations, which in the case of Titan probably arises from upward-propagating gravity waves in the stratosphere. $^{8}$

Within 1 minute of the first dip in flux, the far-limb refracted stellar image appears in both occultations. The central portion of the occultation of the eastern star is marked by longer period and more intense brightenings of the near-limb refracted image than that for the western star. The far-limb images fluctuate in a similar fashion, though uncorrelated and generally with a lower amplitude. At no time in either occultation can we confidently identify a third stellar image, suggesting that Palomar Observatory lay too far from the centerline of either shadow track to fall within the zone of the central flash.

We calculate the occultation midpoint times and impact parameters of the two stars with respect to Titan's geometric center by fitting the simplest possible occultation model to the measured stellar positions. To first order, Titan can simply be considered an occulting circular disk on whose limb refracted stellar images will appear radially outward of the actual position of an occulted star. Such a simple model does not correctly predict stellar positions during the ingress and egress phases of the occultations. Nevertheless, the midpoints and impact parameters can be precisely determined, due to the symmetry of each occultation about its midpoint and the accurately known separation and orientation of the stars with respect to Titan direction and rate of motion. The only free parameters in this model are the position of Titan's geometric center with respect to the stellar reference frame at an arbitrary time (or, equivalently, the two impact parameters), and the effective 

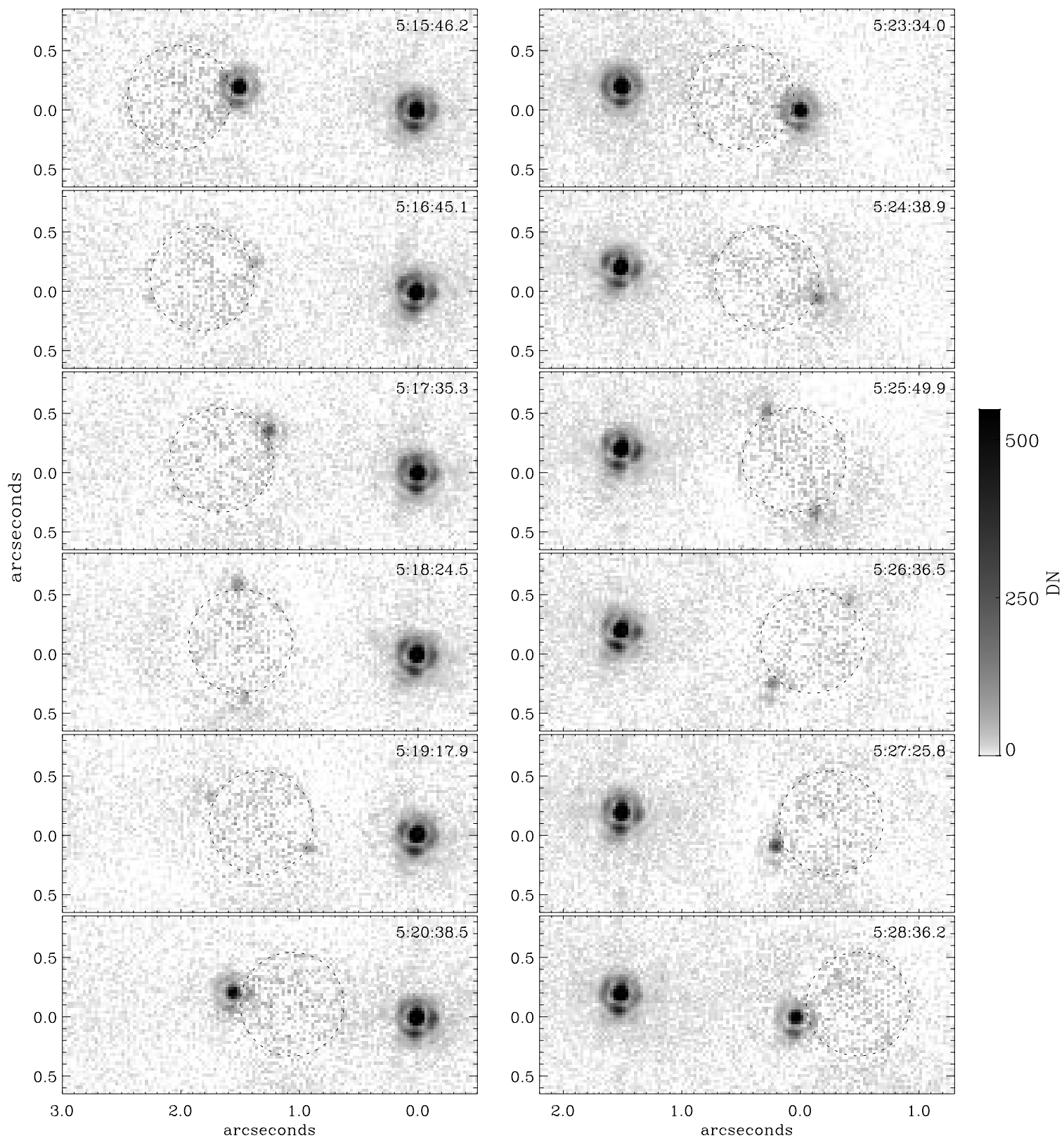

Figure 2. A selection of Titan-subtracted frames from each occultation sequence, chosen to be roughly evenly spaced in time and displayed in a fixed stellar reference frame. The location of Titan's solid limb is illustrated by a dashed line. Refracted stellar images can be seen above both limbs, fluctuating in intensity. 


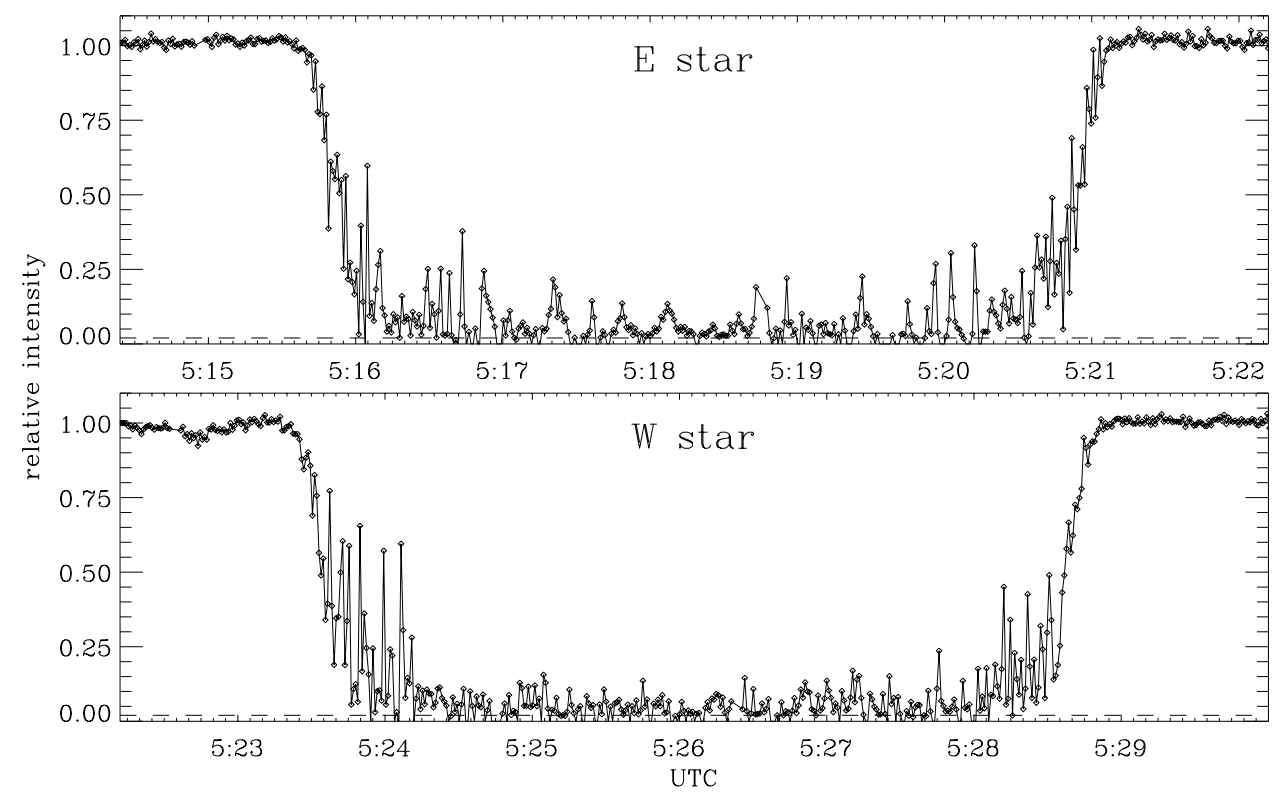

Figure 3. Normalized intensity of the near-limb refracted stellar image, for the two successive occultations. The minimum detectable intensity imposed by our PSF-fitting technique and the photon noise due to Titan is marked with a dashed line.

radius of Titan's occulting disk. We minimized the square of the difference between the measured and predicted stellar predictions in all 4700 frames, weighting by the inverse of the uncertainty in the measured positions, and solved for these three parameters. The derived impact parameters (see Table 1) confirm that Palomar Observatory did not pass sufficiently close to the shadow center of either star to view the central flash. For Titan, this zone extends only $\sim 100 \mathrm{~km}$ from the shadow center. ${ }^{7}$

In Fig 4, we have plotted the measured location of the stars with respect to Titan's center. Some deficiencies of the above model are clear. The apparent altitude of the stellar images above Titan's limb is not constant, but varies from over 300 to $200 \pm 20 \mathrm{~km}$, reaching a clear minimum over each pole, at deepest occultation of each star. These apparent altitudes are only an upper limit to the true maximum depth of penetration of the ray, yet they are significantly lower than the $260-280 \mathrm{~km}$ altitude of deepest penetration predicted using typical models of Titan's stratospheric temperature structure. ${ }^{7}$

We can also use the simple occulting disk model of Titan to test whether surfaces of constant pressure in Titan's atmosphere deviate significantly from spherically symmetric. Fig. 5 presents the position angle (measured from celestial north counter-clockwise) of the near-limb stellar images with respect to the modelderived position of Titan's center as a function of time. To a first approximation, the measured position angles follow those predicted by the circular disk model. Near the midpoints of both occultations, however, slight deviations can be found, and these have been highlighted in the lower panels of Fig. 5 by subtracting the model prediction from the measured position angles. During both occultations, the refracted stellar images appear accelerate ahead of the predicted positions $\sim 30 \mathrm{~s}$ before the midpoint time, slow dramatically during $10 \mathrm{~s}$ centered on the midoint, then accelerate again down the eastern limb of Titan. This phenomenon is more pronounced in the deeper occultation of the eastern star.

The sense in which the measured position angles deviate from those predicted by a spherically symmetric model indicates that the starlight is sampling a region of Titan's stratosphere in which surfaces of constant pressure are oblate. Furthermore, the oblate figure is observed most strongly at mid-latitudes, sampled within $30 \mathrm{~s}$ of the occultation midpoints. This is consistent with the detailed analysis of the $28 \mathrm{Sgr}$ central flashes by Hubbard et. al., ${ }^{7}$ who concluded that surfaces of constant pressure at mid- to high latitude were rendered 


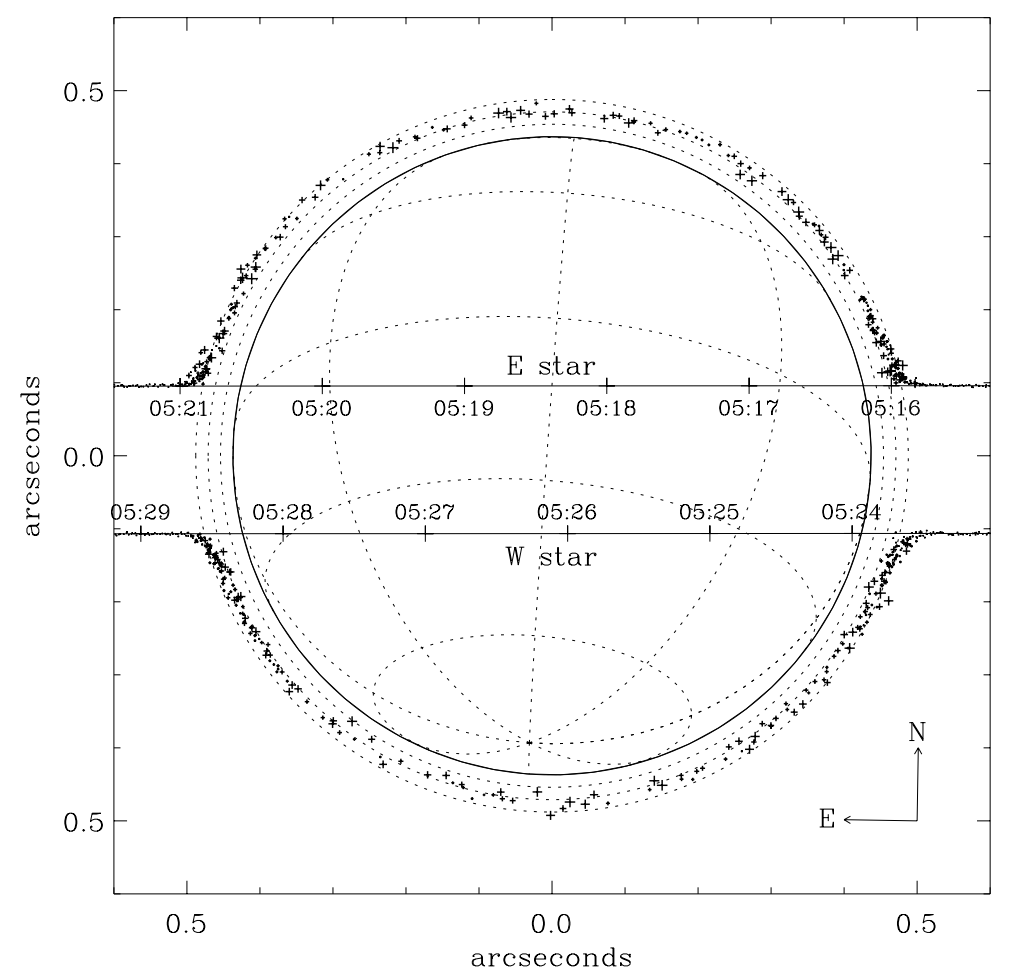

Figure 4. Measured positions of the near-limb refracted stellar images, with respect to Titan's center, for both occultations. The size of the crosses indicates the $1 \sigma$ uncertainty in relative position. Titan's solid surface is represented with lines of latitude and longitude spaced every $30^{\circ}$. Dashed lines above the solid limb indicated apparent altitudes of 100 , 200 , and $300 \mathrm{~km}$. The $200-300 \mathrm{~km}$ altitude at which starlight is refracted through the atmosphere is clearly resolved.

oblate by strong circumpolar winds. A more detailed analysis of the positions of both the near- and far-limb refracted stellar images of each occultation may allow us to map Titan's stratospheric zonal wind velocities at two altitudes in each hemisphere.

\section{CONCLUSIONS}

The high angular resolution achievable with adaptive optics on large-aperture telescopes is revolutionizing many areas of observational planetary science. The double occultation by Titan described in this paper was clearly an unusual event, particularly the convenient geometry and nearly equal brightness of the two stars. Furthermore, the benign atmospheric conditions on the night of the event and the flawless operation of both the AO system and near-infrared camera throughout the event combined to produce a truly remarkable set of images of a unique astronomical phenomenon.

This paper only describes a first glance at the dataset. A more thorough analysis of the positions of the refracted stellar images, both near- and far-limb, should yield and accurate estimate of the zonal wind speeds in both Titan's summer and winter hemispheres. Comparison of their intensities may also provide a direct measure of the opacity of the detached haze layer over both hemispheres. Finally, a reconciliation will have to be reached between our measurements of surprisingly deep penetration into Titan's atmosphere by the stellar light rays and the standard models of Titan's stratospheric structure. 

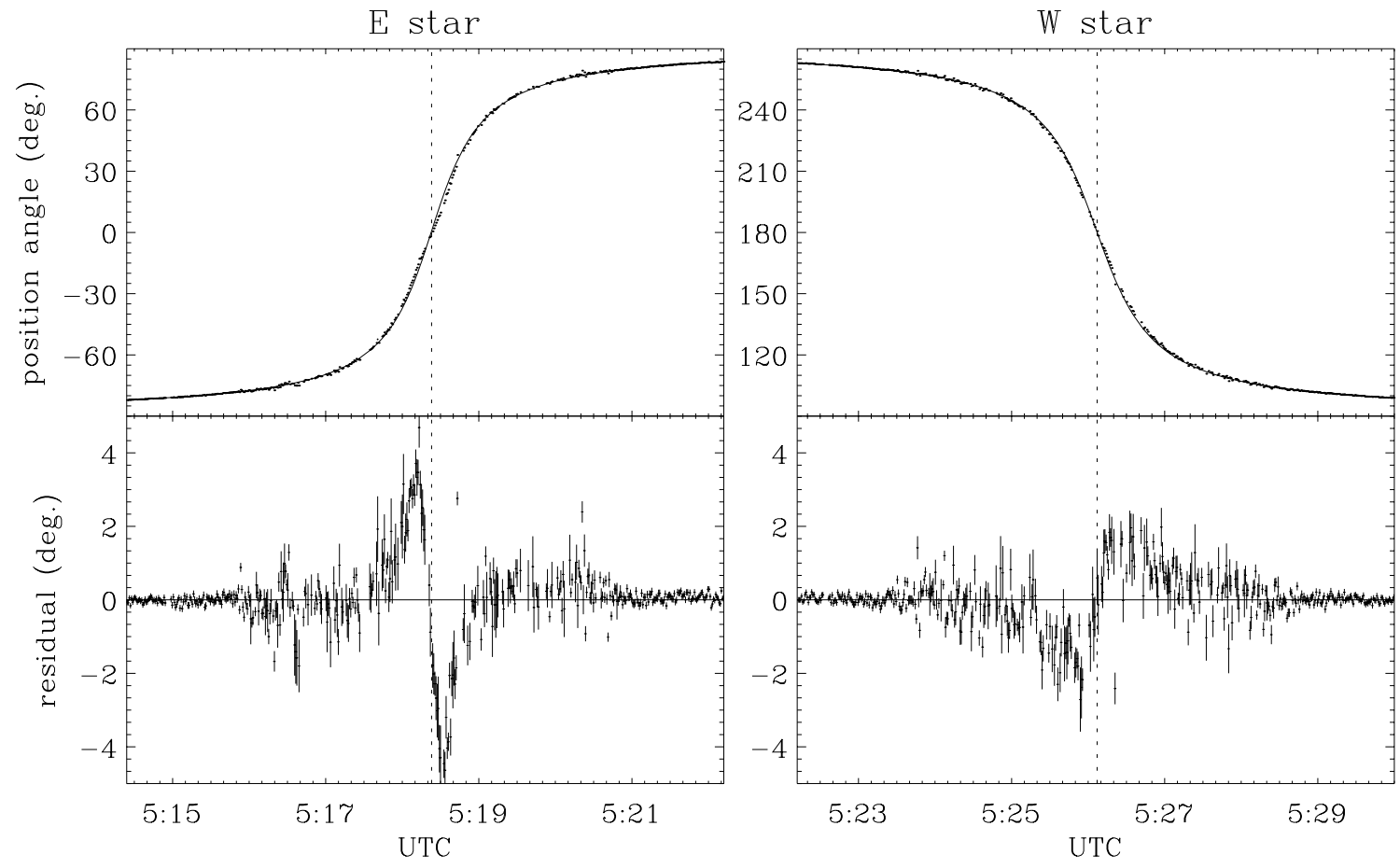

Figure 5. upper panels: Position angles of the near-limb refracted stellar images with respect to Titan's geometric center, as a function of time. A spherically symmetric model (line) and measured values (points near line) agree closely through most of both occultations. lower panels: Measured position angles minus model, highlighting a significant deviation near the occultation midpoints (marked by the vertical dotted line). The stellar images first lead, then lag behind the model, the characteristic signature of high velocity zonal winds in Titan's mid-latitude stratosphere.

\section{ACKNOWLEDGMENTS}

We are grateful to Leslie Young for motivating these observations and providing astrometric and flux predictions. We thank Jean Mueller for able assistance at the controls of the Palomar Hale telescope, and Tom Hayward for help configuring the PHARO camera for these unusual observations. A.H.B. is supported by an NSF Graduate Research Fellowship.

\section{REFERENCES}

1. J. L. Elliot and C. Olkin, "Probing planetary atmospheres with stellar occultations," Ann. Rev. Earth and Planet. Sci. 24, pp. 89-123, 1996.

2. W. A. Baum and A. D. Code, "A photometric observations of the occultation of $\sigma$ Arietis by Jupiter," Astron. J. 58, pp. 108-112, 1953.

3. R. G. French, J. L. Elliot, and P. J. Gierasch, "Analysis of stellar occultation data. effects of photon noise and initial conditions," Icarus 33, pp. 186-202, 1978.

4. J. L. Elliot, R. G. French, E. Dunham, P. J. Gierash, J. Veverka, C. Church, and C. Sagan, "Occultation of $\epsilon$ Geminorum by Mars. II. the structure and extinction of the martian upper atmosphere," Astroph. J. 217, pp. 661-679, 1977.

5. V. R. Eshleman, G. L. Tyler, and W. T. Freeman, "Deep radio occultations and "evolute flashes"; their characteristics and utility for planetary studies," Icarus 37, pp. 612-626, 1979.

6. P. D. Nicholson, C. A. McGhee, and R. G. French, "Saturn's central flash from the 3 July 1989 occultation of 28 Sgr," Icarus 113, pp. 57-83, 1995. 
7. W. B. Hubbard, B. Siccardy, R. Miles, A. J. Hollis, R. W. Forrest, I. K. M. Nicholson, G. Appleby, W. Beisker, C. Bittner, H.-J. Bode, M. Bruns, H. Denzau, M. Nezel, E. Riedel, H. Struckmann, J. Arlot, F. Roques, F. Sevre, W. Thuillot, M. Hoffmann, E. H. Geyer, C. Buil, F. Colas, J. Lecacheux, A. Klotz, E. Thouvenot, J. L. Vidal, E. Carreira, F. Rossi, C. Blanco, S. Cristaldi, Y. Nevo, H. J. Reitsema, N. Brosch, K. Cernis, K. Zdanavicius, L. H. Wasserman, D. Hunten, D. Gautier, E. Lellouch, R. V. Yelle, B. Rizk, F. M. Flasar, C. C. Porco, D. Toublanc, and G. Corugedo, "The occulation of 28 Sgr by Titan," Astron. and Astroph. 269, pp. 541-563, 1993.

8. B. Sicardy, F. Ferri, F. Roques, J. Lecacheux, S. Pau, N. Brosch, Y. Nevo, W. B. Hubbard, H. J. Reitsema, C. Blanco, E. Carreira, W. Beisker, C. Bittner, H.-J. Bode, M. Bruns, H. Denzau, M. Nezel, E. Riedel, H. Struckmann, G. Appleby, R. W. Forrest, I. K. M. Nicolson, A. J. Hollis, and R. Miles, "The structure of Titan's stratosphere from the 28 Sgr occultation," Icarus 142, pp. 357-390, 1999.

9. L. A. Young, http://www.boulder.swri.edu/ layoung/occl/occl.html, 2001.

10. R. G. Dekany, "The Palomar adaptive optics system," in Adaptive optics, D. Optical Society of America, Washington, ed., OSA Technical Digest Series 13, pp. 40-42, 1996.

11. T. L. Hayward, B. Brandl, B. Pirger, C. Blacken, G. E. Gull, J. Schoenwald, and J. R. Houck, "PHARO a near-infrared vamera for the Palomar adaptive optics system," Proc. Astron. Soc. Pac. 113, pp. 103-118, 2001.

12. S. M. Jefferies and J. C. Christou, "Restoration of astronomical images by iterative blind deconvolution," Astroph. J. 415, pp. 862-874, 1993. 